\title{
Hearing others' pain: neural activity related to empathy
}

\author{
Simone Lang • Tao Yu • Alexandra Markl • \\ Friedemann Müller $\cdot$ Boris Kotchoubey
}

Published online: 1 May 2011

(C) Psychonomic Society, Inc. 2011

\begin{abstract}
The human voice is one of the principal conveyers of social and affective communication. Recent neuroimaging studies have suggested that observing pain in others activates neural representations similar to those from the first-hand experience of pain; however, studies on pain expressions in the auditory channel are lacking. We conducted a functional magnetic resonance imaging study to examine brain responses to emotional exclamations of others' pain. The control condition comprised positive (e.g., laughing) or negative (e.g., snoring) stimuli of the human voice that were not associated with pain and suffering. Compared to these control stimuli, pain-related exclamations elicited increased activation in the superior and middle temporal gyri, left insula, secondary somatosensory cortices, thalamus, and right cerebellum, as well as deactivation in the anterior cingulate cortex. The left anterior insular and thalamic activations correlated significantly with the Empathic Concern subscale of the Interpersonal Reactivity Index. Thus, the brain regions involved in hearing others' pain are similar to those activated in the empathic processing
\end{abstract}

S. Lang $(\bowtie)$

Institute of Psychology, Department of Clinical Psychology

and Psychotherapy, University of Heidelberg,

Hauptstrasse 47-51,

69117 Heidelberg, Germany

e-mail: simone.lang@googlemail.com

T. Yu $\cdot$ A. Markl $\cdot$ B. Kotchoubey

Institute of Medical and Behavioral Neurobiology,

University of Tübingen,

Tübingen, Germany

A. Markl $\cdot$ F. Müller

Clinics of Neurological Rehabilitation,

Bad Aibling, Germany of visual stimuli. Additionally, the findings emphasise the modulating role of interindividual differences in affective empathy.

Keywords Empathy $\cdot$ Pain $\cdot$ fMRI $\cdot$ Somatosensory cortex

Empathy is a complex and multidimensional construct that entails not only sharing the emotional experience of another person but also a number of cognitive functions, such as the capacity to understand the other's feelings (Davis, 1996; Decety \& Jackson, 2004; Preston \& de Waal, 2002). The ability to empathize with others who suffer from either psychological or physical pain is critical for maintaining relationships and engaging in prosocial behaviour.

In recent years, the idea that perception-action links in the brain enable us to understand others, a claim that was originally established in the sensorimotor domain, has been expanded to feelings and sensations (Decety \& Lamm, 2006; De Vignemont \& Singer, 2006; Gallese, 2003; Preston \& de Waal, 2002). According to the perceptionaction model of Preston and de Waal, perception and action are represented in shared brain networks, and thus the observation of another person's emotional state automatically activates the observer's representations of that state. These shared neural circuits between self and other prompt the observer to resonate with the emotional state of others (Adolphs, 2002; Preston \& de Waal, 2002).

Previous neuroimaging studies on empathy have mainly focused on shared representations between self and others with respect to such basic emotions as pain or disgust. Several studies have shown that the perception of pain in others activates only the affective components of the pain matrix, such as the anterior cingulate cortex (ACC) and insula (Botvinick et al., 2005; Saarela et al., 2007; Singer et al., 2004). For instance, in the study of Saarela et al., participants 
viewed emotional facial expressions of disgust or pain. The observation of others' pain was related to increased activity in the anterior insula and the ACC. A very similar pattern of activation was found in the study of Singer et al. (2004), in which participants received painrelated stimuli and observed signals indicating that their partners, who were present in the same room, had received the same stimuli.

In contrast, other studies have found evidence for the important role of the sensory dimension of empathy for pain - in particular, when our attention is directed to the somatosensory aspects of the pain experience (Avenanti, Bueti, Galati, \& Aglioti, 2005; Avenanti, Minio-Paluello, Bufalari, \& Aglioti, 2006; Gu \& Han, 2007; Lamm, Nusbaum, Meltzoff, \& Decety, 2007b; Singer et al., 2006). In the study of Lamm, Nusbaum, et al., increased activation in the ACC, anterior insula, parietal cortex, and somatosensory cortex was elicited during watching of photographs displaying painful needle injections of unknown actors. These findings indicate that both the affective and the sensory parts of the pain matrix can be involved in empathy for pain. Likewise, the observation of needles deeply penetrating the hand of a stranger was associated with activity in the midcingulate cortex (MCC), secondary somatosensory cortex, and parietal cortex, but no activation was found in the insula (Costantini, Galati, Romani, \& Aglioti, 2008).

As in many similar instances, controversial data might partially be attributed to interindividual differences in the ability to empathize. Recent evidence suggested that there are two distinct systems for empathy: a basic emotional contagion system and a more advanced, cognitive perspective-taking system. Evidence for a double dissociation between "cognitive empathy" and "emotional empathy" comes from studies of neuropsychological patients (Blair, 2005; Shamay-Tsoory, AharonPeretz, \& Perry, 2008). Individual differences in neural responses to empathic stimuli were found to correlate with behavioural trait measures of empathy in empathy questionnaires, such as the Interpersonal Reactivity Index (IRI; Davis, 1983). The IRI subdivides empathy into the four subscales, "Empathic Concern," "Personal Distress," "Perspective Taking," and "Fantasy." For instance, Singer et al. (2004) found positive correlations between the IRI Empathic Concern subscale, which assesses the affective component of empathy, and left insula and ACC activations. Costantini et al. (2008) reported a negative bilateral correlation between the IRI Perspective Taking subscale, which refers to cognitive empathy, and the activity of secondary somatosensory cortex. The authors interpreted their results as an attempt to control the risk of actually feeling pain by reducing neural activity in the sensorimotor structures involved in pain processing.
The studies cited above used only visual stimuli (e.g., pictures). In real life, empathic responses are frequently elicited by auditory (e.g., laughing, crying) stimuli. Using emotionally charged exclamations is, therefore, another way to assess empathy. The human voice is one of the principal conveyers of social and affective communication. Nonlinguistic vocalizations are a primary means of communication that appear early in ontogenesis: Newborns express and understand emotions vocally long before they learn language (see, e.g., Barr, Hopkins, \& Green, 2000). From a phylogenetic point of view, such vocalizations represent one of the most important means to communicate for most primates, and especially to express affective states (Fichtel \& Hammerschmidt, 2003; Rendall, 2003).

Hearing emotional exclamations of other persons presumes processing of affective prosody, which expresses the emotions of the speaker (Bostanov \& Kotchoubey, 2004; Scherer, 1986). Whereas linguistic prosody, which specifies the semantic and syntactic structure of a sentence, largely involves the rhythm, rate, and pitch, affective prosody, conveying the information of the emotional state of the speaker, mostly relies on voice quality defined on rather brief (tens to hundreds of milliseconds) intervals (Scherer, 1986). Studies examining brain responses to crying and laughing (Sander, Frome, \& Scheich, 2007; Sander \& Scheich, 2001, 2005) have found increased activation in the amygdala, insula, and auditory cortex. In addition, infant crying activated the anterior and posterior cingulate cortices in mothers (Lorberbaum et al., 1999; Lorberbaum et al., 2002). The ability to identify an emotion from prosodic cues is often impaired in neurological patients with rightbrain damage (Ross, Thompson, \& Yenkosky, 1997) and psychiatric disorders such as schizophrenia (Ross et al., 2001), alcoholism (Monnot, Nixon, Lovallo, \& Ross, 2001), depression (Emerson, Harrison, \& Everhart, 1999), and attention-deficit hyperactivity disorder (Manassis, Tannock, \& Barbosa, 2000).

The aim of the present study was to develop painrelated stimulation, which could be applied in neurologic patients with severe alterations in consciousness, such as the vegetative state or minimally conscious state, who are presumably unable to analyse the complex visual scenes usually employed in empathy experiments. For this, we tested whether the perception-action model can be extended to empathy for pain-related exclamations. We hypothesized that neural representations of the "pain matrix" similar to those in the visual context are activated during hearing of pain in others. Furthermore, we also assumed that the auditory cortex, which is involved in the processing of emotional prosody, is activated to emotional exclamations for pain. Finally, we examined interindividual differences in trait empathy using the IRI. 


\section{Method}

Participants

A total of 25 healthy, right-handed volunteers originally participated in the study. The data of 3 were dismissed due to artefacts. The remaining 22 ( 13 female, 9 male) were between 21 and 34 years of age $(M=26.24$ years, $S D=4.6)$. Participants gave informed written consent and were paid for their participation. No participant had any history of neurological or psychiatric illness. The study was approved by the ethical committee of the University of Tübingen and conducted in accordance with the Declaration of Helsinki.

\section{Material and procedure}

Standardised auditory stimuli were used from the International Affective Digitized Sounds (IADS; Stevenson \& James, 2008), which is a database of 111 sounds characterised along the affective dimensions of valence, arousal, and dominance. The experimental stimuli (ES) were selected on the basis that they scored highly in pain and empathy. To test this, 19 healthy participants (who did not participate in the fMRI experiment) evaluated the sounds of human voices from the IADS on being or not painful/empathic. Ten exclamations that, on the basis of this evaluation, maximally expressed pain and suffering were chosen as the experimental stimuli ((ES; sounds) 261, 276, 277, 278, 279, 285, 286, 290, 292, and 423). The ES were characterized by negative valence (mean $2.4 \pm 0.94$ ), high arousal (mean $7.1 \pm 0.86$ ), high pain (mean $7.00 \pm 1.41$ ), and high empathy (mean $7.4 \pm$ 1.01). All stimuli involved screaming, and some of them additionally included a short blow or knocking to a crying individual. Another ten sounds, in which the same properties were manifested in the least degree, were used as control stimuli (CS; Sounds 110, 206, 220, 221, 226, 230, 252, 262, 270 , and 802). The CS contained laughing, singing, snoring, or yawning and were characterised by slightly positive (on average) valence (mean $6.6 \pm 1.24$ ), moderate arousal (mean $4.9 \pm 1.30)$, no pain $(0.23 \pm 0.27)$, and moderate empathy $(4.76 \pm 1.17)$. The stimuli of the two conditions were matched in sound intensity ( 81 vs. $78 \mathrm{~dB}$ for ES and CS, respectively; $t=0.27)$ and fundamental frequency (perceived pitch) (653 vs. $509 \mathrm{~Hz}$ for ES and CS, respectively; $t=1.09$ ).

The sounds (6-s duration each) were presented in 10 blocks of $30 \mathrm{~s}$ each, with a 1.5 -s interstimulus interval and a 30-s baseline between the blocks. Each block comprised four different stimuli in one condition. The different blocks were alternated in a fixed sequence, with each category presented five times. After the experiment, participants were asked to rate the emotional and neutral voices regarding valence and arousal using the SAM rating scale, which ranges from 1 (not aroused or very unpleasant) to 9 (very aroused or very unpleasant). Participants were instructed to listen to the stimuli carefully but not explicitly requested to empathise with the pain of the actors.

Participants heard all auditory stimuli via MRI-compatible headphones with efficient gradient noise suppression (up to $45 \mathrm{~dB}$ ) and a filter system with more than $90 \mathrm{~dB}$ radio frequency suppression. The task sequence was controlled by a PC running the Presentation software (Neurobehavioral Systems, Albany, CA, USA).

Measures of trait empathy

Participants were asked to fill in the German version (Paulus, 2009) of the IRI (Davis 1983, 1996), a 28 -item self-report survey. The IRI is the most widely used self-report measure of dispositional empathy and consists of four subscales: (1) Empathic Concern (which assesses the tendency to experience feelings of sympathy and compassion for others in need), (2) Personal Distress (which assesses the extent to which an individual feels distress as a result of witnessing another's emotional distress) (3) Perspective Taking (which assesses the dispositional tendency of an individual to adopt the perspective of another), and (4) Fantasy (which assesses an individual's propensity to become imaginatively involved with fictional characters and situations). Current social psychological interpretations of the different subscales posit that Empathic Concern and Personal Distress refer to the affective components of empathy, whereas Perspective Taking and Fantasy refer to the cognitive components.

Data analysis of the subjective ratings and the IRI

The subjective ratings and the IRI subscales were analysed using $t$ tests.

\section{fMRI data acquisition and analyses}

MRI data were acquired on a 3-T Siemens Trio System equipped with a 12 channel head coil. Changes in blood oxygenation level dependent (BOLD) T2* weighted magnetic resonance signal were measured using a gradient echo-planar imaging sequence $(\mathrm{TR}=2,380 \mathrm{~ms}, \mathrm{TE}=25 \mathrm{~ms}$, FoV $=$ $210 \mathrm{~mm}$, flip angle $=90^{\circ}, 64 \times 64$ matrix, 40 slices covering the whole brain, slice thickness $3 \mathrm{~mm}$, no gap, voxel size $3.3 \times$ $3.3 \times 3.0 \mathrm{~mm}$ ). A T1-weighted anatomical image was additionally acquired for each participant to allow for anatomical localisation $(\mathrm{TR}=2,300 \mathrm{~ms}, \mathrm{TE}=2.98 \mathrm{~ms}, 160$ slices, voxel size $1.0 \times 1.0 \times 1.1 \mathrm{~mm}$ ). The MATLAB R2010a and SPM8 (Wellcome Department of Imaging Neuroscience, London, U.K.; www.fil.ion.ucl.ac.uk/spm) software was used for data analysis. Each acquisition included 255 volumes. Preprocessing included realignment, coregistration, segmentation, and spatial normalisation (to the template of the 
Montreal Neurological Institute). Then, a Gaussian filter of $8 \mathrm{~mm}$ full width at half maximum was applied to smooth the data spatially. For the statistical analysis of regional differences in brain activation, ES, CS, and baseline (BL) were input into a categorical general linear model (GLM) design at the participant level (Friston et al., 1995). Contrasts between different conditions (ES-BL $>$ CS-BL) were computed for each participant. In the second-level analysis, a one-sample $t$ test was used to obtain an activation pattern for each group. The probability threshold was set at $p<.05$, corrected for family-wise errors (FWE). The minimum cluster extent $(K)$ was set at 20 contiguous voxels. Based on our a priori hypotheses, we additionally conducted a region-of-interest (ROI) analysis for the ACC, insula, primary and secondary somatosensory cortices (SI, SII), thalamus, and cerebellum using automated anatomical labelling masks (TzourioMazoyer et al., 2002) and the WFU Pickatlas (Maldjian, Laurienti, Kraft, \& Burdette, 2003). Clusters of activation were identified with a global height threshold of $p<.05$ (FWE) and an extent threshold of $k>20$ voxels. FWE correction was chosen because it provides a more conservative approach than the false discovery rate (FDR) correction that uses an adaptive threshold based upon both the number of tests and the distribution of the uncorrected $p$ values of those tests (Logan \& Rowe, 2004). Xjview (www.alivelearn. net-(xjview), an SPM viewer toolbox, was used to view and generate the sectional images.

\section{Correlation analyses}

To account for interindividual differences, we computed correlations between differential neural activity, subjective ratings (arousal, valence), and the Interpersonal Reactivity Index subscales. We selected only those brain regions whose activations attained significance. For these brain regions (insula, thalamus, ACC, superior temporal gyrus [STG], middle temporal gyrus [MTG], and cerebellum), masks were created by means of the WFU Pickatlas (Maldijian et al., 2003). We used the "rfxplot" toolbox (Gläscher, 2009) implemented in SPM8 to extract regional BOLD percentage signal changes during pain-related exclamations with respect to the neutral condition. The data were transferred into SPSS and used to calculate Pearson correlations for each region. Given the four IRI indices and the 12 brain ROIs, a total of 48 correlations were obtained. To consider possible alpha inflation due to multiple comparisons, the method of Brien, Venables, James, and Mayo (1984) was used, which appears to be the most powerful of several methods suggested to check the significance of a correlation matrix (Silver \& Dunlap, 1989). The test resulted in a value of $\chi^{2}=8.62$ $(d f=1, p<.005)$. Therefore, the null hypothesis that significant correlations in the matrix were obtained by chance can be regarded as highly improbable.
To account for the number of voxels, we additionally performed a regression analyses with the four IRI subscales as regressors.

\section{Results}

Behavioral results and the Interpersonal Reactivity Index

The mean values of the IRI subscales were $16.73 \pm 3.86$ for "Perspective Taking," $14.27 \pm 5.09$ for "Fantasy," $17.91 \pm$ 3.12 for "Empathic Concern," and $13.41 \pm 3.98$ for "Personal Distress." Pain-related exclamations (ES) were rated as significantly more arousing and more unpleasant than the CS (arousal, $t=4.31, p=.001$; valence, $t=9.85, p<.001$ ). The difference in ratings between the different stimulus sets can be seen in Fig. 1.

Imaging results

As Table 1 and Figs. 2 and 3 show, the ES-CS contrast revealed significant activations in the left and right STG, left and right MTG, bilateral SII, thalamus, left insula, left anterior insula, and right cerebellum. An inverse pattern of activation was obtained in the ACC, whose BOLD signal was significantly weaker to the ES than to the CS. To further analyse this unexpected finding, two additional contrasts-ES versus rest and CS versus rest-were calculated. Whereas no significant difference in the ACC region was found between the CS and the rest conditions, there was a significant deactivation to the ES as compared with rest.

Relationship with subjective ratings and empathy questionnaire

The IRI Empathic Concern subscale was directly related to activity of the left anterior insula $(r=.551, p=.008)$ and the left and right thalamus (left, $r=.504, p=.017$; right, $r=.431$,

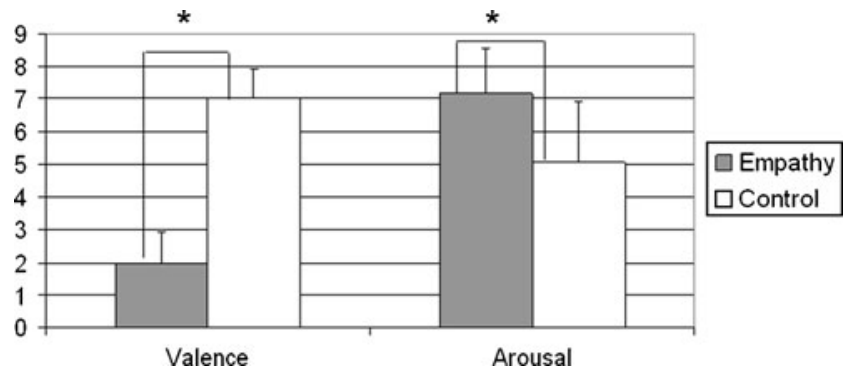

Fig. 1 Subjective ratings of valence $(1=$ very unpleasant, $9=$ very pleasant $)$ and arousal $(1=$ not aroused, $9=$ highly aroused $)$ for the experimental (Empathy) and control stimuli. The asterisks indicate significant differences 
Table 1 Clusters of significant signal change (activation and deactivation) in the right $(\mathrm{R})$ and left $(\mathrm{L})$ hemispheres

\begin{tabular}{llrrrrrr}
\hline & & & \multicolumn{5}{c}{ MNI Coordinates } \\
\cline { 5 - 6 } Brain Area & L/R & BA & $k$ & $x$ & $y$ & $z$ & T \\
\hline Activation & & & & & & & \\
STG & $\mathrm{L}$ & 22 & 566 & -57 & -40 & 16 & 5.61 \\
STG & $\mathrm{R}$ & 41 & 474 & 54 & -40 & 22 & 7.18 \\
MTG & $\mathrm{L}$ & 21 & 147 & -54 & -43 & 10 & 5.85 \\
MTG & $\mathrm{R}$ & 22 & 63 & 48 & -37 & 4 & 5.41 \\
SII & $\mathrm{L}$ & 40 & 56 & -60 & -43 & 25 & 6.24 \\
SII & $\mathrm{R}$ & 40 & 56 & 54 & -49 & 22 & 5.58 \\
SMG & $\mathrm{R}$ & 40 & 95 & 60 & -40 & 25 & 5.92 \\
Insula & $\mathrm{L}$ & 13 & 144 & -36 & 11 & -5 & 4.40 \\
Thalamus & $\mathrm{L}$ & & 74 & -12 & -16 & 7 & 4.05 \\
Thalamus & $\mathrm{R}$ & & 49 & 15 & -16 & 10 & 3.69 \\
Cerebellum & $\mathrm{R}$ & & 117 & 3 & -55 & -5 & 4.32 \\
Deactivation & & & & & & & \\
ACC & $\mathrm{L}$ & 32 & 75 & 35 & -8 & -5 & 3.62 \\
\hline
\end{tabular}

Coordinates reflect positions relative to the Montreal Neurological Institute Atlas (voxel threshold $p<.05$, corrected for family-wise errors). $k=$ number of voxels belonging to clusters; $\mathrm{BA}=$ Brodmann area, $\mathrm{SII}=$ secondary somatosensory cortex, $\mathrm{STG}=$ superior temporal gyrus, $\mathrm{MTG}=$ middle temporal gyrus, $\mathrm{SMG}=$ supramarginal gyrus, $\mathrm{ACC}=$ anterior cingulate cortex.

$p=.045)$. The Personal Distress subscale was inversely related to activation of the left and right cerebellum (left, $r=-.553, p=.008$; right, $r=-.556, p=.006$ ), the left and right STG (left, $r=-.589, p=.004$; right, $r=-.451$ $p=.035$ ), and the left and right MTG (left, $r=-.576, p=$ .005 ; right, $r=-.590, p=.004)$. See Fig. 4 . The arousal and valence ratings did not significantly correlate with brain activations in any region $(p>.10)$.

Significant correlations obtained in the regression analysis are displayed in Table 2.
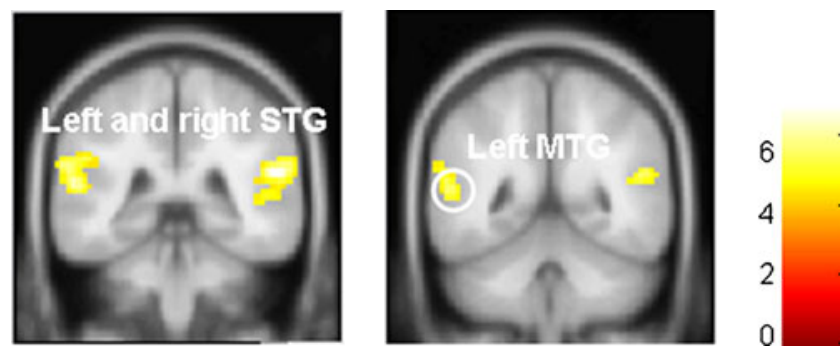

Fig. 2 Significant clusters in the left and right superior temporal gyri (STG) and the left middle temporal gyrus (MTG) from the random-effects contrast of experimental stimuli (ES) versus control stimuli (CS). The color bar indicates t-values. The threshold was $p<.05$, whole-brain corrected for family-wise errors

\section{Discussion}

Brain responses to sounds of pain and suffering

The goal of the present study was to investigate how nonverbal exclamations indicating pain and suffering modulate the neural underpinnings of emotional empathy. The subjective ratings indicated successful induction of the unpleasant affective reactions to pain-related exclamations. The functional imaging data corroborated the behavioural findings, suggesting that hearing others' pain elicited increased activation in brain areas associated with "shared representations." These included the left anterior insula, SII, thalamus, and right cerebellum. Consistent with previous neuroimaging studies using complex visual stimuli, activation of these areas involved in empathy for pain was replicated with pain-related exclamations, without a physical sensation of actual pain (Benuzzi, Luim, Duzzi, Nichelli, \& Porro, 2008; Jackson, Meltzoff, \& Decety, 2005; Jackson, Rainville, \& Decety, 2006; Simon, Craig, Miltner, \& Rainville, 2006).

The involvement of the somatosensory cortex in empathy for pain has been inconsistently reported in the literature. Some studies showed that only the affective component of the pain matrix are involved in empathy for pain, and thus that only emotional, not sensory, representations of pain are shared between self and others (Saarela et al., 2007; Singer et al., 2004). On the other hand, previous transcranial magnetic stimulation studies (Avenanti et al., 2005; Avenanti et al., 2006) and somatosensory evoked potential studies (Bufalari, Aprile, Avenanti, Di Russo, \& Aglioti, 2007) highlighted the possible sensorimotor aspect of empathy for pain. In recent years, subsequent fMRI studies supported these findings, demonstrating that both affective and somatosensory components of the pain matrix are activated during watching of complex visual scenes (Benuzzi et al., 2008; Costantini et al., 2008; Han et al., 2009; Lamm, Batson, \& Decety, 2007a). The present finding indicates a pivotal role of the somatosensory cortex in hearing the pain of other individuals, although participants were not instructed to pay attention to the somatosensory aspect.

In contrast to previous studies using visual stimuli, in the present study we additionally observed a stronger bilateral activation in the STG (BA 22), extending to the Heschl gyrus (BA 41) and the MTG (BAs 21, 22) in both hemispheres, to pain exclamations as compared with control stimuli. Previous studies have shown that the STG and MTG are involved in the processing of speech prosody (Ethofer et al., 2006; Mitchell, Elliott, Barry, Cruttenden, \& Woodruff, 2003; Wiethoff, Wildgruber, Grodd, \& Ethofer, 2009; Wiethoff et al., 2008; Wildgruber et al., 2005). STG activations were also found in studies that investigated crying, laughing, and fear screams (Phillips et al., 1998; Sander \& Scheich, 2001, 2005) and were associated with vocal expressions of fear, presumably 

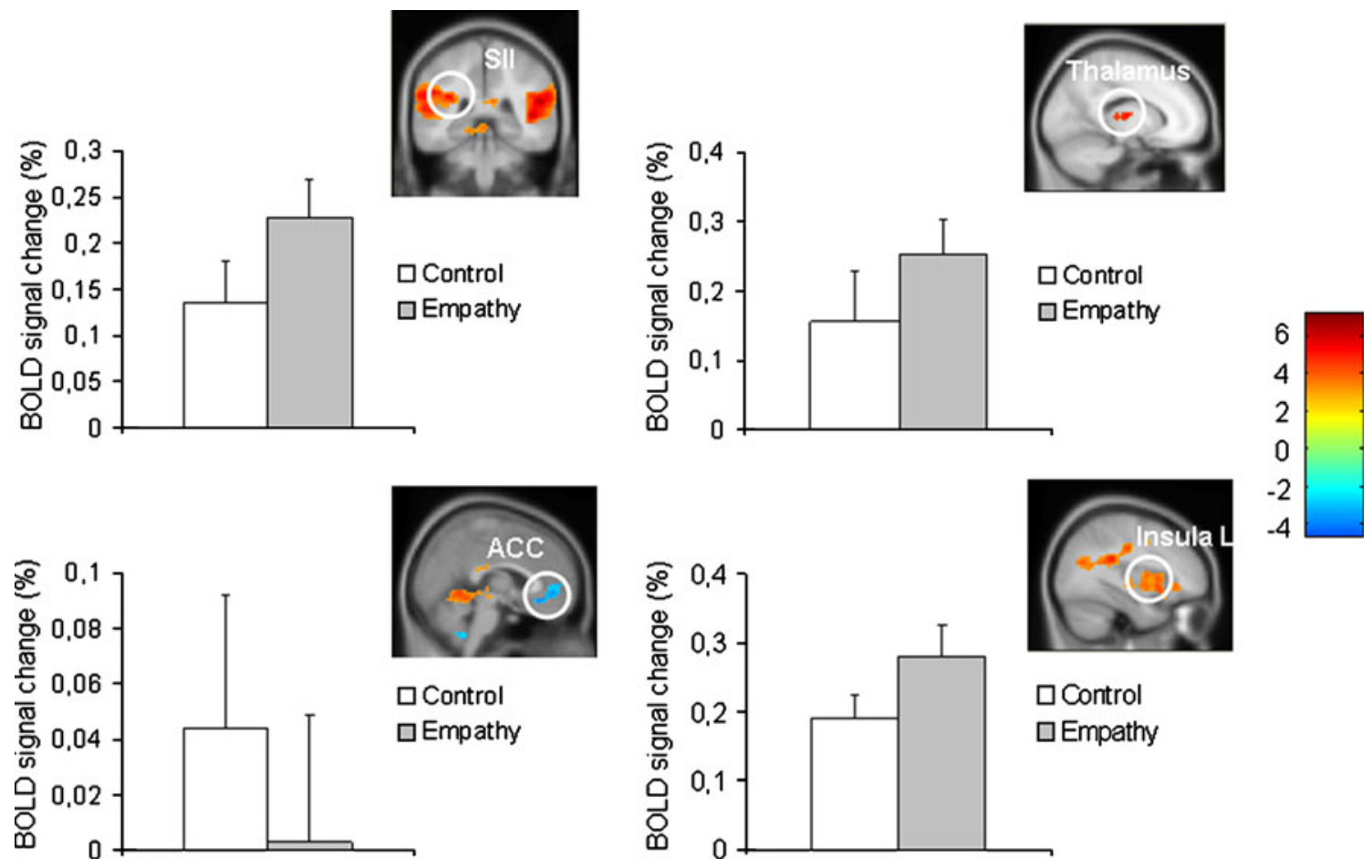

Fig. 3 Significant activation in response to others' pain (Empathy) versus the control condition. The white circles on the brain images indicate the main clusters related to the pain network. The height

threshold for illustrating the clusters was $p<.05$, corrected for familywise errors. SII, secondary sensory cortex; ACC, anterior cingulate cortex
Fig. 4 (a) Correlation between the percentage of BOLD signal change in the left anterior insula and empathic concern, as measured by the Interpersonal Reactivity Index, in the contrast of experimental stimuli (ES) versus control stimuli (CS). (b) Correlation between the percentage of BOLD signal change in the right middle temporal gyrus (MTG) and scores on the Personal Distress subscale in the contrast of ES versus CS. The lines represent the linear best fits; $r$ refers to the coefficient of correlation. Each correlation is significant at the $p<.005$ level, and the whole correlation matrix is significant as well $(p<.005)$

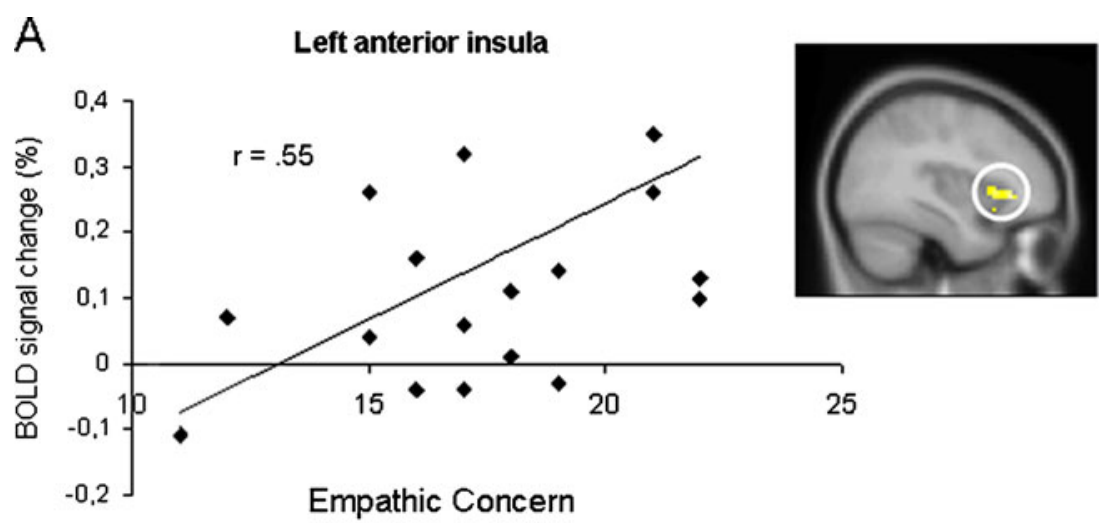

B

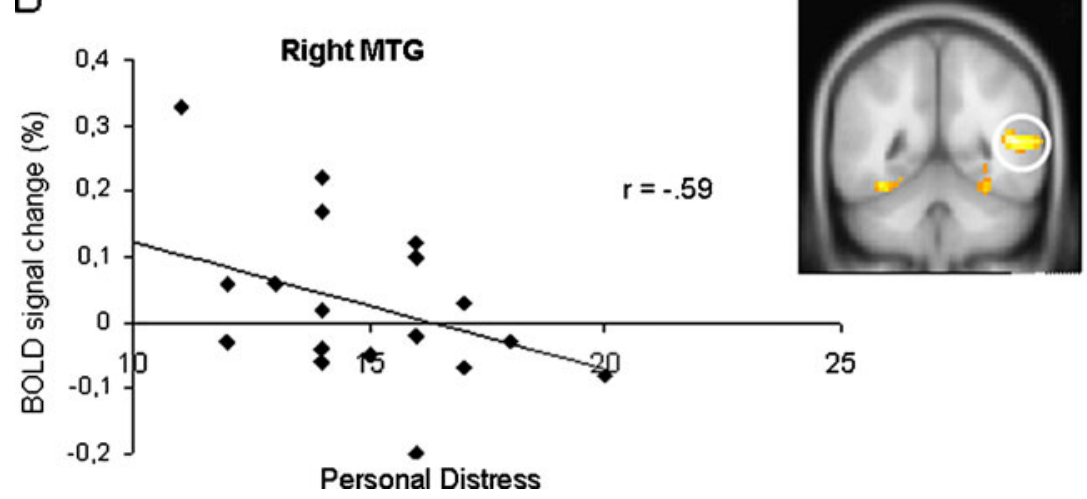


Table 2 Significant correlations between brain areas and the Interpersonal Reactivity Index subscales in the regression analysis of the contrast of experimental versus control stimuli

\begin{tabular}{lllllllll}
\hline & & \multicolumn{7}{c}{ MNI Coordinates } \\
\cline { 5 - 6 } Brain Area & L/R & BA & $k$ & $x$ & $y$ & $z$ & T \\
\hline Empathic Concern & & & & & & & \\
Anterior insula & $\mathrm{L}$ & 13 & 32 & -36 & 17 & 1 & 3.69 \\
Thalamus & $\mathrm{R}$ & & 28 & 12 & -13 & 16 & 3.72 \\
Personal Distress & & & & & & & \\
STG & $\mathrm{L}$ & 22 & 13 & -51 & -28 & 7 & 5.08 \\
STG & $\mathrm{R}$ & 21 & 10 & 63 & -1 & -5 & 4.61 \\
STG & $\mathrm{R}$ & 21 & 24 & 60 & 2 & -8 & 4.91 \\
STG & $\mathrm{R}$ & 13 & 37 & 44 & -19 & 10 & 6.04 \\
MTG & $\mathrm{R}$ & 39 & 23 & 51 & -70 & 10 & 4.25 \\
Cerebellum & $\mathrm{L}$ & & 131 & -9 & -85 & -17 & 4.29 \\
\hline
\end{tabular}

indicating a relationship to the extraction of emotional content from vocal stimuli (Zhang, Shu, Zhou, Wang, \& $\mathrm{Li}, 2010)$. Moreover, the STG and MTG are typically engaged in cognitive empathy (Carrington \& Bailey, 2009; Völlm et al., 2006). Also, greater involvement of the STG and MTG has been found in empathy studies using cuebased paradigms in which participants receive and observe stimuli related to pain in others (Lamm, Decety, \& Singer, 2011). Both mechanisms - that is, deciphering auditory emotional features and cognitive components of empathymight be supposed to have operated in the present experiment.

Surprisingly, we found a deactivation of the ACC. The ACC has been implicated in response to threatening stimuli (Peyron, Laurent, \& García-Larrea, 2000), empathy for pain (Budell, Jackson, \& Rainville, 2010; Singer et al., 2004), and vocal expressions of fear (Phillips et al., 1998). Although the ACC could function as a part of the so-called default system of the brain, its deactivation in the present experiment can hardly be related to the default system, because the activity to control stimuli was not decreased as compared with rest. Recently, Han et al. (2009) compared empathic neural responses to pictures of pain delivered against a background of neutral, painful, or happy faces. Observation of pain pictures together with pain faces reduced ACC activity, while the activity of the secondary somatosensory cortex increased. The authors suggested that extensive emotional arousal may result in a decrease of ACC activity. In this vein, the deactivation in the ACC to emotional exclamations in the present experiment might result from the highly arousing emotional context induced by the blocked design. However, the exact functional meaning of BOLD signal decreases is not known, and therefore the finding should be interpreted with caution.

As expected, the ES differed from the CS in terms of arousal and valence, and thus one might ask whether the neurophysiological results could be ascribed to those aspects of arousal and valence only, without being attributed to the subjective feeling of empathy. This would be a behaviorist interpretation. We do not think that this option can be ruled out completely, because whatever empathic stimuli we use, they would necessarily have some physical and emotional properties distinct from those of nonempathic stimuli. The same holds true, of course, for visual stimuli, since any control picture has to possess some physical and emotional features that differ from those of empathic pictures. The hypothesis of empathic specificity would be fortified if the same stimuli did not elicit the same neural activations in individuals clinically unable to experience empathic feelings (e.g., severe psychopaths), but again, the stimuli would probably be less arousing and unpleasant for such individuals than for the normal population.

However, though an exact proof of empathic specificity is impossible, the indirect evidence is in line with this hypothesis. The pattern of activations obtained in the present study (as well as that obtained in the previous visual studies) is more in accord with the pain matrix than with activations usually obtained in studies of highly arousing negative emotions such as anxiety and anger. Particularly, the activation of the sensory subsystem of pain (e.g., SII) is difficult to explain in response merely to negative emotion. Further evidence for the involvement of empathic experience can be drawn from the correlations with the IRI data (see below).

Correlations between brain activation and subjective reports

A complex pattern of correlations between self-report data and different brain activations was found. The positive correlation between the left anterior insula and the IRI Empathic Concern subscale is in line with Singer et al. (2004), who also observed higher left (but not right) insular activity in participants with higher Empathic Concern scores. Additionally, the activity of the left and right thalamus in response to the ES also correlated positively with the Empathic Concern scores. Like the insula, the thalamus is a limbic brain area that is involved in emotional processing. Increased thalamic activation was observed during visual perception of threat (Lane et al., 1997). Also, Nummenmaa, Hirvonen, Parkkola, and Hietanen (2008) found increased activation in the thalamus in response to emotional versus cognitive empathic stimuli. Empathic Concern is assumed to be an index of emotional empathy, assessing the tendency to experience feelings of sympathy and compassion for others in need. The results highlight an important role of the thalamus in the empathic experience related to pain.

Negative correlations were found between the Personal Distress subscale and activations in the left and right cerebellum and bilateral STG and MTG. Personal Distress involves experiencing the distress of another individual as 
if it is one's own, which is related to a collapse of the selfother distance. In developmental science, it is generally considered a primitive form of empathic response. because infants imitate the emotional distress of others without awareness of the other's situation or condition (Decety, 2007; Eisenberg, 2000; Lamm et al., 2007b). Davis (1996) noted that personal distress is characterized by a negative affective tone and self-oriented thought processes. Individuals with higher experienced personal distress tend to be more anxious and uncomfortable, regardless of the states of mind of others. Thus, it can be speculated that participants who show lower activation in the STG, cerebellum, and MTG are those with a higher distress withdrawal reaction. Notably, we did not find significant correlations between SII activity and any subjective measure. This lack of correlation may possibly indicate that the SII activation, related to the sensory rather than the emotional subsystem of the pain matrix, is independent of the individual differences in dispositional empathy. Also importantly, neither arousal nor valence significantly correlated with any brain activation. This result would be surprising if we attribute the activations to unspecific negative emotions elicited by pain-related stimuli. However, the result is in line with the idea that the activations are specifically related to the experience of empathy.

\section{Limitations and perspectives}

This is the first study of empathic brain responses to higharousal auditory pain stimuli carried out on a relatively small sample. The most important limitation is our (as well as all other authors') failure to find the optimal control condition, in which the stimuli presented are acoustically similar and possess the same arousal and valence ratings, but do not elicit an empathic first-person experience. For example, Benuzzi et al. (2008) used disgusting pictures as controls for painrelated pictures but found that disgust was significantly less unpleasant than pain; thus, the difference in neural responses (i.e., empathy vs. disgust) could also be attributed to the different valence. We did not find appropriate auditory disgust stimuli, and thus do not know whether such stimuli might attain the level of negative valence characteristic for pain-related cries. Even if they do, the difference in arousal would possibly remain. Since there is no reason to believe that emotional brain structures respond independently to arousal and valence, even a perfect control of one of these variables would make the control insufficient as long as the other remained different. Although control stimuli of similar arousal and valence could be found among technical sounds (e.g., a jackhammer or some war-related sounds), these are so different from the tones of humans voice that they also could not count as good controls. Because of the lack of an appropriate control condition, we cannot completely rule out the possibility that the brain activations in the present study are related to differences in arousal or valence rather than empathy. Further, the present study included both male and female volunteers. A recent study (Schulte-Rüther, Markowitsch, Shah, Fink, \& Piefke, 2008) demonstrated that females recruited areas containing mirror neurons to a higher degree than males. Further studies should pay attention to gender differences in the brain networks related to empathy.

\section{Conclusions}

The results replicate and considerably extend previous neuroimaging studies of empathy for watching pain in others, showing responses of almost the entire pain matrix in response to exclamations manifesting pain and suffering-including not only emotional but also sensory components of pain (Costantini et al., 2008; Lamm et al., 2007b). Thus, the data provide evidence in the auditory domain for the perceptionaction model, postulating that perceiving or imagining another person in a particular emotional state automatically activates a representation of this state in the observer. Moreover, we showed that this presumably bottom-up process is modulated by interindividual differences in the ability to empathise with others. The presented experiment also has a possible practical value: Because emotional exclamations are a primary means of communication, since they appear early in both onto- and phylogenesis, these stimuli might be applied to testing empathy for pain in neurological patients with severely impaired cognition and consciousness - for instance, those in a vegetative state, with akinetic mutism, or in a minimally conscious state (Kotchoubey \& Lang, 2011).

Author note This study was supported by the German Research Foundation (Deutsche Forschungsgemeinschaft, Ko-1753/10).

\section{References}

Adolphs, R. (2002). Recognizing emotion from facial expressions: Psychological and neurological mechanisms. Behavioral and Cognitive Neuroscience Review, 1, 21-62.

Avenanti, A., Bueti, D., Galati, G., \& Aglioti, S. M. (2005). Transcranial magnetic stimulation highlights the sensorimotor side of empathy for pain. Nature Neuroscience, 8, 955-960.

Avenanti, A., Minio-Paluello, I., Bufalari, I., \& Aglioti, S. M. (2006). Stimulus-driven modulation of motor-evoked potentials during observation of others' pain. Neuroimage, 32, 316-324.

Barr, R. G., Hopkins, B., \& Green, J. A. (2000). Crying as a sign, a symptom, and a signal: Clinical, emotional and developmental aspects of infant and toddler crying (Clinics in Developmental Medicine, No. 152). London: Mac Keith Press.

Benuzzi, F., Luim, F., Duzzi, D., Nichelli, P. F., \& Porro, C. A. (2008). Does it look painful or disgusting? Ask your parietal and cingulate cortex. The Journal of Neuroscience, 23, 923-931. 
Blair, R. J. R. (2005). Responding to the emotions of others: Dissociating forms of empathy through the study of typical and psychiatric populations. Consciousness and Cognition, 14, 698718. doi:10.1016/j.concog.2005.06.004.

Bostanov, V., \& Kotchoubey, B. (2004). Recognition of affective prosody: Continuous wavelet measures of event-related brain potentials to emotional exclamations. Psychophysiology, 41, 259-268. doi:10.1111/j.1469-8986.2003.00142.x.

Botvinick, M., Jha, A. P., Bylsma, L. M., Fabian, S. A., Solomon, P. E., \& Prkachin, K. M. (2005). Viewing facial expressions of pain engages cortical areas involved in the direct experience of pain. Neuroimage, 25, 312-319.

Brien, C. J., Venables, W. N., James, A. T., \& Mayo, O. (1984). An analysis of correlation matrices. Biometrika, 71, 545-554.

Budell, L., Jackson, P., \& Rainville, P. (2010). Brain responses to facial expressions of pain: Emotional or motor mirroring? Neuroimage, 53, 355-363. doi:10.1016/j.neuroimage.2010.05.037.

Bufalari, I., Aprile, T., Avenanti, A., Di Russo, F., \& Aglioti, S. M. (2007). Empathy for pain and touch in the human somatosensory cortex. Cerebral Cortex, 17, 2553-2561.

Carrington, S. J., \& Bailey, A. J. (2009). Are there theory of mind regions in the brain? A review of the neuroimaging literature. Human Brain Mapping, 30, 2313-2335. doi:10.1002/hbm.20671.

Costantini, M., Galati, G., Romani, G. L., \& Aglioti, S. M. (2008). Empathic neural reactivity to noxious stimuli delivered to body parts and non-corporeal objects. The European Journal of Neuroscience, 28, 1222-1230. doi:10.1111/j.1460-9568.2008.06406.x.

Davis, M. H. (1983). Measuring individual differences in empathy: Evidence for a multidimensional approach. Journal of Personality and Social Psychology, 44, 113-126.

Davis, M. H. (1996). Empathy: A social psychological approach. Boulder, CO: Westview Press.

De Vignemont, F., \& Singer, T. (2006). The empathic brain: How, when and why? Trends in Cognitive Sciences, 10, 435-441.

Decety, J. (2007). A social cognitive neuroscience model of human empathy. In E. Harmon-Jones \& P. Winkielman (Eds.), Social neuroscience: Integrating biological and psychological explanations of social behavior. New York: Guilford Press.

Decety, J., \& Jackson, P. L. (2004). The functional architecture of human empathy. Behavioral and Cognitive Neuroscience Review, 3, 71-100.

Decety, J., \& Lamm, C. (2006). Human empathy through the lens of social neuroscience. Scientific World Journal, 20, 1146-1163.

Eisenberg, N. (2000). Emotion, regulation, and moral development. Annual Review of Psychology, 51, 665-697.

Emerson, C. S., Harrison, D. W., \& Everhart, D. E. (1999). Investigation of receptive affective prosodic ability in schoolaged boys with and without depression. Neuropsychiatry, Neuropsychology, and Behavioral Neurology, 12, 102-109.

Ethofer, T., Anders, S., Erb, M., Herbert, C., Wiethoff, S., Kissler, J., et al. (2006). Cerebral pathways in processing of affective prosody: A dynamic causal modeling study. Neuroimage, 30, 580-587.

Fichtel, C., \& Hammerschmidt, K. (2003). Responses of squirrel monkeys to their experimentally modified mobbing calls. The Journal of the Acoustical Society of America, 113, 2927-2932. doi:10.1121/1.1548158.

Friston, K. J., Holmes, A. P., Worsley, K. J., Poline, J. B., Frith, C. D., \& Frackowiak, R. S. J. (1995). Statistical parametric maps in functional imaging: A general linear approach. Human Brain Mapping, 2, 189-210.

Gallese, V. (2003). The roots of empathy: The shared manifold hypothesis and the neural basis of intersubjectivity. Psychopathology, 36, 171-180.

Gläscher, J. (2009). Visualization of group inference data in functional neuroimaging. Neuroinformatics, 7, 72-83.
Gu, X., \& Han, S. (2007). Attention and reality constraints on the neural processes of empathy for pain. Neuroimage, 36, 256267.

Han, S., Fan, Y., Xu, X., Qin, J., Wu, B., Wang, X., et al. (2009). Empathic neural responses to others' pain are modulated by emotional contexts. Human Brain Mapping, 30, 3227-3237.

Jackson, P. L., Meltzoff, A. N., \& Decety, J. (2005). How do we perceive the pain of others? A window into the neural processes involved in empathy. Neuroimage, 24, 771-779.

Jackson, P. L., Rainville, P., \& Decety, J. (2006). To what extent do we share the pain of others? Insight from the neural bases of pain empathy. Pain, 125, 5-9.

Kotchoubey, B., \& Lang, S. (2011). Intuitive versus theory-based assessment of consciousness: The problem of low-level consciousness. Clinical Neurophysiology, 22, 430-432.

Lamm, C., Batson, C. D., \& Decety, J. (2007a). The neural substrate of human empathy: Effects of perspective-taking and cognitive appraisal. Journal of Cognitive Neuroscience, 19, 42-58. doi:10.1162/jocn.2007.19.1.42.

Lamm, C., Nusbaum, H. C., Meltzoff, A. N., \& Decety, J. (2007b). What are you feeling? Using functional magnetic resonance imaging to assess the modulation of sensory and affective responses during empathy for pain. PLoS ONE, 2, e1292.

Lamm, C., Decety, J., \& Singer, T. (2011). Meta-analytic evidence for common and distinct neural networks associated with directly experienced pain and empathy for pain. Neuroimage, 54, 24922502. doi:10.1016/j.neuroimage.2010.10.014

Lane, R. D., Reiman, E. M., Bradley, M. M., Lang, P. J., Ahern, G. L., Davidson, R. J., et al. (1997). Neuroanatomical correlates of pleasant and unpleasant emotion. Neuropsychologia, 35, 1437 1444.

Logan, B. R., \& Rowe, D. B. (2004). An evaluation of thresholding techniques in fMRI analysis. Neuroimage, 22, 95-108.

Lorberbaum, J. P., Newman, J. D., Dubno, J. R., Horwitz, A. R., Nahas, Z., Teneback, C. C., et al. (1999). Feasibility of using fMRI to study mothers responding to infant cries. Depression and Anxiety, 10, 99-104.

Lorberbaum, J. P., Newman, J. D., Horwitz, A. R., Dubno, J. R., Lydiard, R. B., Hamner, M. B., et al. (2002). A potential role for thalamocingulate circuitry in human maternal behavior. Biological Psychiatry, 51, 431-445. doi:10.1016/S0006-3223(01) 01284-7.

Maldjian, J. A., Laurienti, P. J., Kraft, R. A., \& Burdette, J. H. (2003). An automated method for neuroanatomic and cytoarchitectonic atlas-based interrogation of fMRI data sets. NeuroImage, 19, $1233-1239$.

Manassis, K., Tannock, R., \& Barbosa, J. (2000). Dichotic listening and response inhibition in children with comorbid anxiety disorders and ADHD. Journal of American Academy of Child and Adolescence Psychiatry, 39, 1152-1159.

Mitchell, R. L., Elliott, R., Barry, M., Cruttenden, A., \& Woodruff, P. W. (2003). The neural response to emotional prosody, as revealed by functional magnetic resonance imaging. Neuropsychologia, $41,1410-1421$

Monnot, M., Nixon, S., Lovallo, W., \& Ross, E. (2001). Altered emotional perception in alcoholics: Deficits in affective prosody comprehension. Alcoholism, Clinical and Experimental Research, 25, 362-369.

Nummenmaa, L., Hirvonen, J., Parkkola, R., \& Hietanen, J. K. (2008). Is emotional contagion special? An fMRI study on neural systems for affective and cognitive empathy. Neuroimage, 43, $571-580$.

Paulus, C. (2009). Der Saarbrücker Persönlichkeitsfragebogen (IRI) zur Messung von Empathie. Psychometrische Evaluation der deutschen Version des Interpersonal Reactivity Index. Available at http://psydok.sulb.uni-saarland.de/volltexte/2009/2363/. 
Peyron, R., Laurent, B., \& García-Larrea, L. (2000). Functional imaging of brain responses to pain: A review and meta-analysis. Neurophysiogie Clinique, 30, 263-288.

Phillips, M. L., Young, A. W., Scott, S. K., Calder, A. J., Andrew, C., Giampietro, V., et al. (1998). Neural responses to facial and vocal expressions of fear and disgust. Proceedings of the Royal Society $B, 265,1809-1817$.

Preston, S. D., \& de Waal, F. B. M. (2002). Empathy: Its ultimate and proximate bases. Behavioral Brain Science, 25, 1-72.

Rendall, D. (2003). Acoustic correlates of caller identity and affect intensity in the vowel-like grunt vocalizations of baboons. The Journal of the Acoustical Society of America, 113, 3390-3402.

Ross, E. D., Orbelo, D. M., Cartwright, J., Hansel, S., Burgard, M., Testa, J. A., et al. (2001). Affective-prosodic deficits in schizophrenia: Profiles of patients with brain damage and comparison with relation to schizophrenic symptoms. Journal of Neurology, Neurosurgery and Psychiatry, 70, 597-604.

Ross, E. D., Thompson, R. D., \& Yenkosky, J. (1997). Lateralization of affective prosody in brain and the callosal integration of hemispheric language functions. Brain and Language, 56, 27-54.

Saarela, M. V., Hlushchuk, Y., Williams, A. C. C., Schurmann, M., Kalso, E., \& Hari, R. (2007). The compassionate brain: Humans detect intensity of pain from another's face. Cerebral Cortex, 17, 230-237.

Sander, K., Frome, Y., \& Scheich, H. (2007). FMRI activations of amygdala, cingulate cortex, and auditory cortex by infant laughing and crying. Human Brain Mapping, 28, 1007-1022. doi:10.1002/hbm.20333.

Sander, K., \& Scheich, H. (2001). Auditory perception of laughing and crying activates human amygdala regardless of attentional state. Cognitive Brain Research, 12, 181-198.

Sander, K., \& Scheich, H. (2005). Left auditory cortex and amygdala, but right insula dominance for human laughing and crying. Journal of Cognitive Neuroscience, 17, 1519-1531. doi:10.1162/ 089892905774597227.

Scherer, K. R. (1986). Vocal affect expression: A review and a model for future research. Psychological Bulletin, 99, 143-165.

Schulte-Rüther, M., Markowitsch, H. J., Shah, N. J., Fink, G. R., \& Piefke, M. (2008). Gender differences in brain networks supporting empathy. Neuroimage, 42, 393-403.

Shamay-Tsoory, S. G., Aharon-Peretz, J., \& Perry, D. (2008). Two systems for empathy: A double dissociation between emotional and cognitive empathy in inferior frontal gyrus versus ventromedial prefrontal lesions. Brain, 132, 617-627. doi:10.1093/brain/awn279.

Silver, N. C., \& Dunlap, W. P. (1989). A Monte Carlo study of testing the significance of correlation matrices. Educational and Psychological Measurements, 49, 563-569.

Simon, D., Craig, K. D., Miltner, W. H., \& Rainville, P. (2006). Brain responses to dynamic facial expressions of pain. Pain, 126, 309318.

Singer, T., Seymour, B., O’Doherty, J., Kaube, H., Dolan, R. J., \& Frith, C. D. (2004). Empathy for pain involves the affective but not sensory components of pain. Science, 303, 1157-1161. doi:10.1126/science.1093535.

Singer, T., Seymour, B., O’Doherty, J. P., Stephan, K. E., Dolan, R. J., \& Frith, C. D. (2006). Empathic neural responses are modulated by the perceived fairness of others. Nature, 439, 466-469. doi:10.1038/nature04271.

Stevenson, R. A., \& James, T. W. (2008). Affective auditory stimuli: Characterization of the International Affective Digitized Sounds (IADS) by discrete emotional categories. Behavior Research Methods, 40, 315-321. doi:10.3758/BRM.40.1.315.

Tzourio-Mazoyer, N., Landeau, B., Papathanassiou, D., Crivello, F., Etard, O., Delcroix, N., et al. (2002). Automated anatomical labeling of activations in SPM using a macroscopic anatomical parcellation of the MNI MRI single-subject brain. Neuroimage, 15, 273-289.

Völlm, B. A., Taylor, A. N. W., Richardson, P., Corcoran, R., Stirling, J., McKie, S., et al. (2006). Neuronal correlates of theory of mind and empathy: A functional magnetic resonance imaging study in a nonverbal task. Neuroimage, 29, 90-98.

Wiethoff, S., Wildgruber, D., Grodd, W., \& Ethofer, T. (2009). Response and habituation of the amygdala during processing of emotional prosody. Neuroreport, 20, 1356-1360.

Wiethoff, S., Wildgruber, D., Kreifelts, B., Becker, H., Herbert, C., Grodd, W., et al. (2008). Cerebral processing of emotional prosody-Influence of acoustic parameters and arousal. Neuroimage, 39, 885-893.

Wildgruber, D., Riecker, A., Hertrich, I., Erb, M., Grodd, W., Ethofer, T., et al. (2005). Identification of emotional intonation evaluated by fMRI. Neuroimage, 24, 1233-1241.

Zhang, L., Shu, H., Zhou, F., Wang, X., \& Li, P. (2010). Common and distinct neural substrates for the perception of speech rhythm and intonation. Human Brain Mapping, 31, 1106-1116. 